\title{
Dobesilate for dry age-related macular degeneration*
}

\author{
Pedro Cuevas $^{1 \#, ~ L u i s ~ A . ~ O u t e i r i n ̃ o ~}{ }^{2}$, Carlos Azanza ${ }^{2}$, Javier Angulo ${ }^{1}$, Guillermo Giménez-Gallego ${ }^{3}$ \\ ${ }^{1}$ Departamento de Investigación, IRYCIS, Hospital Universitario Ramón y Cajal, Madrid, Spain \\ ${ }^{2}$ Departamento de Oftalmología, Hospital de Día Pío XII, Madrid, Spain \\ ${ }^{3}$ Departamento de Estructura y Función de Proteínas, Centro de Investigaciones Biológicas, CSIC, Madrid, Spain \\ Email: " pedro.cuevas@ hrc.es \\ Received 24 March 2013; revised 29 August 2013; accepted 16 September 2013 \\ Copyright (C) 2013 Pedro Cuevas et al. This is an open access article distributed under the Creative Commons Attribution License, \\ which permits unrestricted use, distribution, and reproduction in any medium, provided the original work is properly cited.
}

\begin{abstract}
We have evaluated the effects of intravitreal dobesilate, a synthetic fibroblast growth factor inhibitor, in patients with dry age-related macular degeneration, an inflammatory-related retinal disease without available treatment up to date. 36 eyes from 36 patients with dry age-related macular degeneration were treated with a single intravitreal dobesilate injection. The end points were the improvement from baseline visual acuity and normalization of retinal histology at one month. Intravitreal dobesilate injection resulted in a significant improvement in functional and anatomical outcomes at one month after injection. Our results suggest that intravitreal dobesilate may increase the chance of visual acuity gain in dry age-related macular degeneration, even in cases with initial low vision. This study supports the findings of previously published case reports, regarding the short-term improvement in visual acuity by intravitreal dobesilate injection in different degenerative retinal diseases.
\end{abstract}

Keywords: Dry Age-Related Macular Degeneration; Fibroblast Growth Factor Inhibition; Intravitreal Dobesilate

\section{INTRODUCTION}

Age-related macular degeneration (AMD) is the primary cause of blindness and visual disability in people aged over 50 and its prevalence increases exponentially after the age of $70[1,2]$. AMD is diagnosed as either wet (neovascular) or dry (atrophic). Wet AMD is characterized by choroidal nevoascularization $(\mathrm{CNV})$ : the formation of

\footnotetext{
"Consent: obtained.

Competing interest: the authors report no conflicts of interest in this work.

"Corresponding author.
}

hyperpermeable new blood vessels beneath the retinas that leak plasma and often bleed, leading to the formation of scar tissue which can severely and irreversibly compromise visual acuity. Although it constitutes only $10 \%-15 \%$ of all cases, wet AMD accounts for almost $80 \%$ of AMD-related blindness [3].

Dry AMD, in contrast, does not involve leaking vessels from choroidal vasculature. Dry AMD is characterized by a well-defined constellation of clinical features, including drusen, pigment abnormalities/focal hyper- or hypo-pigmentation of the retinal pigment epithelium (RPE), and geographic atrophy (GA) of the macula. GA represents the atrophic late stage of dry AMD [1,2]. GA is characterized by roughly oval areas of hypopigmentation and is usually the consequence of RPE cell loss. Loss of RPE cells, responsible for the overlying photoreceptors surviving, leads to the gradual degeneration of nearly photoreceptors, resulting in thinning of the retina. RPE degeneration leads to the death of photoreceptor cells causing irreversible vision loss. Since the natural evolution of dry AMD is toward a wet AMD condition, the holy grail of therapy for AMD is to avoid the development of CNV. Today, there are no approved treatments for dry AMD.

Considering the significant medical, personal, social and economic costs of AMD, the need for novel therapeutic and preventive strategies for AMD is pressing. Innovation in AMD pharmacotherapy, in turn, depends largely upon a thorough understanding of the molecular mechanisms underlying AMD pathogenesis. There is ample evidence that inflammation plays an important role in both dry and wet AMD [4-9]. Several studies have documented a significant association between inflammatory diseases and upregulation of fibroblast growth factor (FGF) [10-16]. Recently, it has been posited the importance of FGF in neuroinflammatory diseases such as AMD where inflammation is one of the main components of disease progression and FGF and its receptors (FGF/FGFR) are prominently expressed [17]. 
This study supports an important clinical interest for searching safe and efficient inhibitors of FGF/FGFR axis to treat retinal inflammatory diseases, including AMD. The aim of this study was to analyze treatment with a single dose of intravitreal dobesilate in patients who were diagnosed with dry age-related macular degeneration.

\section{METHODS}

\subsection{Participants}

In this consecutive study, more than 200 patients with age-related macular degeneration who visited the Clínica Oftalmológica Hospital Pío XII of Madrid (Spain) between January 2012 and October 2012, were screened, and 36 eyes from 36 patients with dry age-related macular degeneration were included. Baseline data for all study patients is shown in Table 1. The study was approved by the local institutional review board and informed consent was obtained from every patient for the intravitreal injection. The subjects were also provided information about the off-label use of dobesilate.

\subsection{Inclusion/Exclusion Criteria}

Inclusion criteria were the presence of early, intermediate or late stages of dry AMD. Eyes that met any of the following criteria were excluded from enrolment: 1) had severe disease that was judged by the treating investigator as being unlikely to benefit from further therapy (such as those with central ischemia or macular scarring); 2) had vision loss from other coexisting ocular disease and 3) had undergone ocular surgical interventions within 6 months prior to study entry.

\subsection{Procedures}

Examination at baseline included best corrected visual

Table 1. Baseline characteristics of the patients.

\begin{tabular}{cc}
\hline \multicolumn{1}{c}{ Sex } \\
Male & 16 \\
Female & 20 \\
\hline Mean & $70 \pm 7$ \\
\hline Intermediate phase of dry AMD & 14 \\
Male & 16 \\
Female & \\
\hline Late phase of dry AMD (geographic atrophy) \\
Male \\
Female
\end{tabular}

acuity (BCVA) with a Snellen chart at a distance of 20 feet, slit lamp biomicroscopy of the anterior segment and fundus, and spectral domain optical coherence tomography (SD-OCT). The data recorded included complains as scotoma, blurred vision and metamorphopsia.

The intravitreal injection of dobesilate was performed in accordance with the guidelines for intravitreal injections [18]. Before injection administration, the eye was washed with povidone-iodine (5\%) and the eyelashes and lid region were then wiped, also with povidone-iodine $(5 \%)$. Then, each patient received $18.75 \mathrm{mg}$ of dobesilate in a single intravitreal injection of $150 \mu \mathrm{l}$ of a solution of diethylamonium 2,5-dihydroxybenzesulfonate (etamsylate; dycinone ${ }^{\circledR}$, Sanofi-Aventis, Paris, France). Antibiotic eye drops were then applied. Patients returned to the outpatient clinic for routine postinjection follow-up at day one and day three after injection; a slit lamp examination and pressure measurements were performed to rule out intraocular inflammation or elevated intraocular pressure (IOP). BCVA, slit lamp biomicroscopy of the anterior segment and fundus, and SDCOT were conducted again at 1 month postinjection. The ocular symptoms, such as scotoma, metamorphopsia and blurred vision were examined by questioning each patient before and after treatment. Scotoma and metamorphopsia on the Amsler grid were scored according to Verma et al. [19]. Accordingly, scotoma and metamorphopsia were graded numerically from 0 to IV, where a grade 0 indicates absence of scotoma and metamorhopsia, and a grade IV indicates very severe scotoma and metamorphopsia. Blurred vision was scored from 0 to 4 , where a score of 0 indicates no vision disturbance and a score of 4 indicates very severe blurred vision.

\subsection{Endpoints}

The primary endpoint was the improvement of visual acuity at 1 month compared with baseline. The second endpoint was the effect of treatment in the normalization of retinal structure at the same time after treatment.

\subsection{Statistics}

The paired t-test was used to statistically evaluate changes in visual acuity at baseline and one month after treatment. A $p$ value $<0.05$ was considered to be statistically significant. Graphic representation of the data is expressed as mean \pm standard error of the mean (SEM).

\section{RESULTS}

\subsection{Visual Outcomes}

In the current study, 36 eyes of 36 patients of both sexes were enrolled. All patients tolerated well the injection. The mean BCVA at baseline was $0.44 \pm 0.03$. At one 
month examination, 32 eyes $(89 \%)$ showed visual acuity improvement and only 4 eyes (11\%) experienced visual acuity worsening. Individual patient BCVA values before and after treatment are shown in Table 2. The mean BCVA after treatment was $0.59 \pm 0.03(p<0.001)$. Changes in BCVA after intravitreal injection of dobesilate appear in Figure 1. As the Table 3 shows, scotoma, metamorphopsia and blurred vision were consistently reduced, inducing the improvement of quality of vision in all 32 cases.

\subsection{Anatomical Outcomes}

The effect of intravitreal dobesilate in retinal structural outcomes was assessed with SD-OCT. At baseline, inner retinal layer was normal, whereas the outer retinal layers showed structural alterations: 1) the integrity of photoreceptor inner segment and outer segment were not preserved; 2) retinal pigment epithelium (RPE) showed rarefactions and thinning. In contrast, normalization of outer retinal layers was achieved in 32 patients after dobesilate treatment. As an example of effectiveness of

Table 2. Change of BCVA from baseline to last observation.

\begin{tabular}{|c|c|c|c|c|c|}
\hline $\mathrm{P}$ & Baseline & Treatment & $\mathrm{P}$ & Baseline & Treatment \\
\hline \multicolumn{5}{|c|}{ One month } & One month \\
\hline 1 & 0.40 & 0.50 & 19 & 0.60 & 0.90 \\
\hline 2 & 0.50 & 0.60 & 20 & 0.40 & 0.60 \\
\hline 3 & 0.60 & 0.70 & 21 & 0.50 & 0.70 \\
\hline 4 & 0.50 & 0.80 & 22 & 0.30 & 0.40 \\
\hline 5 & 0.40 & 0.80 & 23 & 0.40 & 0.50 \\
\hline 6 & 0.50 & 0.60 & 24 & 0.20 & 0.60 \\
\hline 7 & 0.60 & 0.60 & 25 & 0.20 & 0.40 \\
\hline 8 & 0.40 & 0.70 & 26 & 0.20 & 0.90 \\
\hline 9 & 0.20 & 0.90 & 27 & 0.30 & 0.60 \\
\hline 10 & 0.60 & 0.20 & 28 & 0.30 & 0.50 \\
\hline 11 & 0.40 & 0.60 & 29 & 0.40 & 0.50 \\
\hline 12 & 0.50 & 0.40 & 30 & 0.50 & 0.60 \\
\hline 13 & 0.60 & 0.50 & 31 & 0.70 & 0.80 \\
\hline 14 & 0.70 & 0.60 & 32 & 0.30 & 0.50 \\
\hline 15 & 0.70 & 0.80 & 33 & 0.20 & 0.30 \\
\hline 16 & 0.70 & 0.80 & 34 & 0.40 & 0.60 \\
\hline 17 & 0.40 & 0.40 & 35 & 0.30 & 0.20 \\
\hline 18 & 0.50 & 0.90 & 36 & 0.30 & 0.50 \\
\hline
\end{tabular}

Intravitreal dobesilate injection improved BCVA in patients with dry agerelated macular degeneration. P: Patient number $(n=36)$.

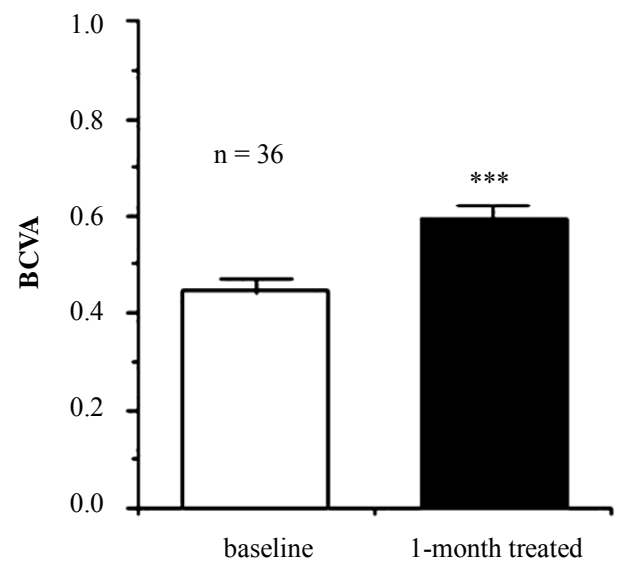

Figure 1. Visual acuity 1 month after an intravitreal injection of dobesilate showing statistically significant improvement.

dobesilate, we show a SD-OCT scan of a patient with GA (Figure 2). GA is characterized by confluent areas of cell death of photoreceptors and RPE, is bilateral in more than half of patients, and is responsible for $10 \%$ of cases of legal blindness from AMD [20]. To date no effective treatment for progressive vision loss is available for GA.

\subsection{Safety}

There were no cases of treatment-associated complications such as retinal detachment, endophthalmitis or persistent elevated IOP.

\section{DISCUSSION}

Clinically and histologically, AMD is generally classified into two major subtypes: dry or non-exudative AMD, of which GA is a severe form, and wet or exudative AMD. Wet AMD is very debilitating and often develops after early dry AMD. The key feature of wet AMD is choroidal neovascularization (CNV), the growth of new hyperpermeable blood vessels from the choroids into the region underlying the RPE or extending into the subretinal space [21]. Dry AMD progresses more slowly and manifests RPE and photoreceptor cell dysfunction and degeneration [4]. Although the aetiology of AMD is not completely understood, it is indisputable that inflammation has a critical role in both dry and wet AMD [4-9]. Consequently, conventional therapies based on inhibiting choroidal neovascularization do not seem to be an appropriate strategy for dry AMD management. However, the aetiology of this disease suggests that the treatment of inflammation could be a suitable alternative to treat dry AMD.

Previously, we have reported the efficacy of dobesilate, a synthetic inhibitor of FGF/FGFR axis [22] in patients with retinal diseases [23-27]. FGF in spite of being an 
Table 3. Change in ocular symptoms score from baseline to last observation.

\begin{tabular}{|c|c|c|c|c|c|c|c|c|c|c|c|c|}
\hline & $\mathbf{P}$ & B & $\mathbf{T}$ & $\mathbf{P}$ & $\mathbf{B}$ & $\mathbf{T}$ & $\mathbf{P}$ & B & $\mathbf{T}$ & $\mathbf{P}$ & B & $\mathbf{T}$ \\
\hline S & 1 & II & I & 10 & II & II & 19 & III & 0 & 28 & II & 0 \\
\hline $\mathbf{M}$ & & II & I & & I & 0 & & II & 0 & & III & I \\
\hline BV & & 1 & 0 & & 1 & 1 & & 1 & 0 & & 3 & 1 \\
\hline S & 2 & III & II & 11 & I & III & 20 & II & I & 29 & II & I \\
\hline $\mathbf{M}$ & & I & I & & I & II & & II & I & & III & 0 \\
\hline BV & & 1 & 0 & & 1 & 2 & & 2 & 1 & & 2 & 0 \\
\hline S & 3 & I & 0 & 12 & I & 0 & 21 & I & 0 & 30 & II & I \\
\hline $\mathbf{M}$ & & I & 0 & & II & 0 & & I & I & & I & 0 \\
\hline BV & & 0 & 0 & & 1 & 0 & & 1 & 0 & & 1 & 0 \\
\hline S & 4 & 0 & 0 & 13 & I & I & 22 & II & I & 31 & 0 & 0 \\
\hline $\mathbf{M}$ & & I & 0 & & I & III & & II & 0 & & 0 & 0 \\
\hline BV & & 1 & 0 & & 0 & 1 & & 1 & 0 & & 1 & 0 \\
\hline S & 5 & I & 0 & 14 & II & II & 23 & I & 0 & 32 & 0 & 0 \\
\hline $\mathbf{M}$ & & 0 & 0 & & I & 0 & & I & I & & 0 & 0 \\
\hline BV & & 1 & 0 & & 1 & 1 & & 1 & 0 & & 1 & 0 \\
\hline S & 6 & I & 0 & 15 & I & II & 24 & II & I & 33 & 0 & 0 \\
\hline $\mathbf{M}$ & & 0 & 0 & & I & II & & II & I & & I & 0 \\
\hline BV & & 0 & 0 & & 1 & 1 & & 1 & 0 & & 1 & 0 \\
\hline S & 7 & I & 0 & 16 & II & I & 25 & III & I & 34 & III & II \\
\hline $\mathbf{M}$ & & 0 & 0 & & I & I & & II & 0 & & III & II \\
\hline BV & & 0 & 0 & & 0 & 1 & & 3 & 0 & & 2 & 2 \\
\hline S & 8 & II & I & 17 & I & 0 & 26 & II & 0 & 35 & I & 0 \\
\hline $\mathbf{M}$ & & II & 0 & & I & 0 & & II & 0 & & I & 0 \\
\hline BV & & 1 & 0 & & 1 & 0 & & 1 & 0 & & 1 & 1 \\
\hline S & 9 & III & 0 & 18 & I & I & 27 & III & I & 36 & II & III \\
\hline $\mathbf{M}$ & & II & 0 & & II & II & & III & I & & II & II \\
\hline BV & & 1 & 0 & & 1 & 1 & & 3 & 0 & & 2 & 2 \\
\hline
\end{tabular}

Dobesilate injection in the vitreous gel improved ocular symptoms associated to dry age-related macular degeneration. S: Scotoma; M: Metamorphopsia; BV: Blurred Vision; P: Patient number $(\mathrm{n}=36)$; B: Baseline; T: after one month of Treatment.

angiogenesis promoter [28] is involved also in inflammation [10-16] and seems to play key roles in neurodegenerative diseases such as Alzheimer's and Parkinson's diseases as well as in AMD [17].

Dobesilate features suggest that its intravitreal application could be of potential clinical benefit in dry AMD management. In this report, we describe the rapid normalization of retinal structure, running parallel to a con-

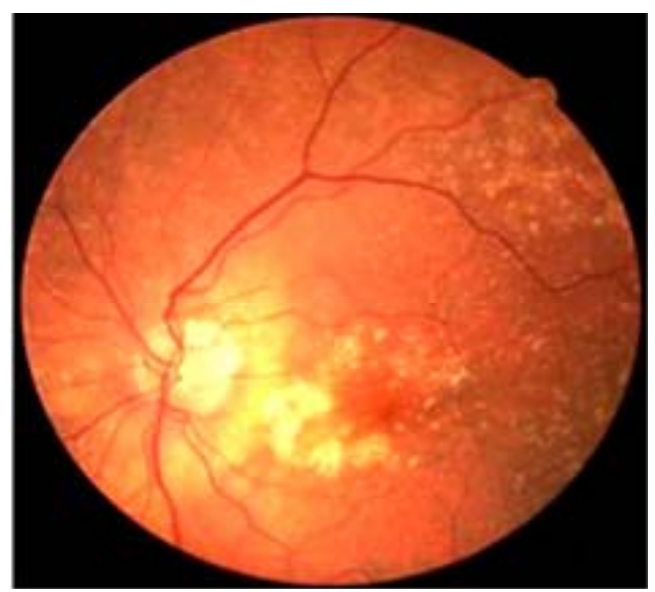

(a)

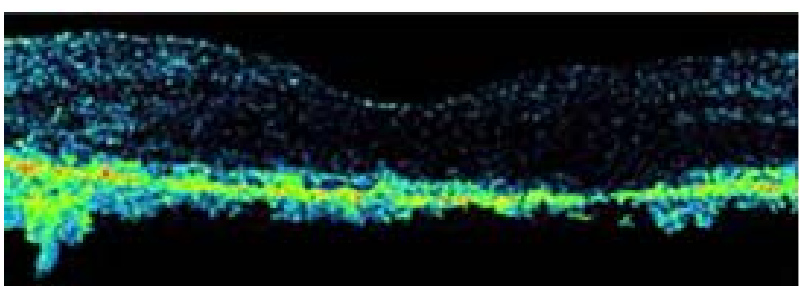

(b)

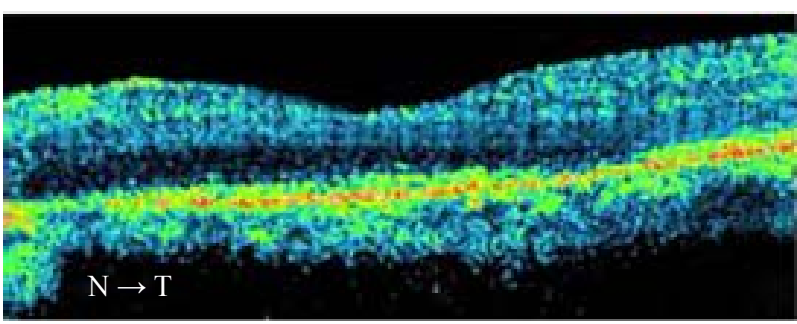

(c)

Figure 2. Fundus photographs of the left eye of an enrolled patient at baseline with peripheral areas of pigment accumulation, autofluorescence deposits and patches of geographic atrophy of the retinal pigment epithelium (a). Optical coherence tomography of the central macula before treatment with dobesilate (b), showing loss in some areas of both outer retinal layers and pigment epithelial cells, and one month after treatment (c), with improvement of vision from 0.20 to 0.60 . Note the anatomical recovery of outer retinal layers and retinal pigment epithelium after a single intravitreal injection of dobesilate.

siderable improvement of visual acuity in patients with dry AMD after a single intravitreal administration of dobesilate. This study adds some more valuable data to the recently reported promising good results to the use of dobesilate in both dry and wet AMD and also in other retinal diseases [23-27].

It may result somehow surprising that an inhibitor of FGF shows the good safety profile of dobesilate, given the broad spectrum of physiological activities in which FGF is involved [29-32]. In effect, FGF has been detected in most adult tissues tightly bound to the sulphated glycosaminoglycans (GAG) of the extracellular matrix 
(ECM), at times at very high levels [22]. Thus, under normal physiological conditions, FGF does not act as a signalling molecule in solution, but as a solid phase growth factor. As far as FGF remains part of the ECM, it cannot be inhibited by dobesilate, which has an affinity constant for FGF approximately 3000 times lower than that of the sulphated GAG of the ECM; furthermore, it reaches extraordinarily high concentrations [22]. Nevertheless, the physiological FGF signalling system gets sometimes subverted, causing very serious physiological disturbances, when high concentrations of free FGF are accumulated, either through uncontrolled synthesis or mobilization by heparanases and other specialized proteins which could be upregulated in inflammatory conditions [33-35]. In contrast to ECM bounded FGF, dobesilate efficiently inhibits free FGF [22]. Consequently, it inhibits mainly pathological FGF, leaving the relatively untouched physiological FGF pool.

Several limitations are inherent in the current study. First, the design was prospective, the sample size was small and there was no control group. Second, this one time follow-up of patients does not describe visual acuity evolution over time and the results may be transient. However, this study shows for the first time the efficacy and safety of a new therapy for dry age-related macular degeneration that has been considered as an orphan disease. Furthermore, treatment with dobesilate increased visual acuity above the expected baseline decrease during the natural evolution of untreated dry AMD. In sum, dobesilate with a long history of use, and abundant preclinical data supporting its biological effects and its potential efficacy, is promising as a FGF targeted therapy for AMD. A large population cohort study is needed to establish the effectiveness of intravitreal dobesilate in treating dry AMD patients.

\section{AUTHOR CONTRIBUTIONS}

Conceived and designed the study: PC, GGG, LO. Performed the study LO, CA. Analyzed the data: LO, CA, JA, GGG, and PC. PC, GGG, wrote the paper. All authors read and approved the final manuscript.

\section{ACKNOWLEDGEMENTS}

We are indebted to the patients who participated in this study.

\section{REFERENCES}

[1] Ambati, J., Ambati, B.K., Yoo, S.H., Ianchulev, S. and Adamis, A.P. (2003) Age-related macular degeneration: Etiology, pathogenesis, and therapeutic strategies. Survey of Ophthalmology, 48, 257-293. http://dx.doi.org/10.1016/S0039-6257(03)00030-4

[2] van Leeuwen, R., Klaver, C.C., Vingerling, J.R., Hofman, A. and de Jong, P.T. (2003) Epidemiology of age-related maculopathy: A review. European Journal of Epidemiology, 18, 845-854.

http://dx.doi.org/10.1023/A:1025643303914

[3] Klein, R., Klein, B.E., Knudtson, M.D., Meuer, S.M., Swift, M. and Gangnon, R.E. (2007) Fifteen-year cumulative incidence of age-related macular degeneration: The beaver dam eye study. Ophthalmology, 114, 253-262. http://dx.doi.org/10.1016/j.ophtha.2006.10.040

[4] Rodrigues, E.B. (2007) Inflammation in dry age-related macular degeneration. Ophthalmologica, 221, 143-152. http://dx.doi.org/10.1159/000099293

[5] Nowak, J.Z. (2006) Age-related macular degeneration (AMD): Pathogenesis and therapy. Pharmacological Reports, 58, 353-363.

[6] Patel, M. and Chan, C.C. (2008) Immunopathological aspects of age-related macular degeneration. Seminars in Immunopathology, 30, 97-110.

http://dx.doi.org/10.1159/000099293

[7] Hollyfield, J.G., Bonilha, V.L., Rayborn, M.E., Yang, X., Shadrach, K.G., Lu, L., Ufret, R.L, Salomon, R.G. and Perez, V.L. (2008) Oxidative damage-induced inflammation initiates age-related macular degeneration. Nature Medicine, 14, 194-198.

http://dx.doi.org/10.1038/nm1709

[8] Buschini, E., Piras, A., Nuzzi, R. and Vercelli, A. (2011) Age related macular degeneration and drusen: Neuroinflammation in the retina. Progress in Neurobiology, 95, 14-25. http://dx.doi.org/10.1016/j.pneurobio.2011.05.011

[9] Telander, D.G. (2011) Inflammation and age-related macular degeneration (AMD). Seminars in Ophthalmology, 26, 192-197.

http://dx.doi.org/10.1016/j.pneurobio.2011.05.011

[10] Byrd, V.M., Ballard, D.W., Miller, G.G. and Thomas, J.W. (1999) Fibroblast growth factor-1 (FGF-1) enhances IL-2 production and nuclear translocation of NF-kappaB in FGF receptor-bearing Jurkat T cells. Journal of Immunology, 162, 5853-5859.

[11] Meij, J.T., Sheikh, F., Jiménez, S.K., Nickerson, P.W., Kardami, E. and Cattini, P.A. (2002) Exacerbation of myocardial injury in transgenic mice overexpressing FGF2 is T cell dependent. American Journal of PhysiologyHeart and Circulatory Physiology, 282, H547-H555.

[12] Rossini, M., Cheunsuchon, B., Donnert, E., Ma, L.J., Thomas, J.W., Neilson, E.G. and Fogo, A.B. (2005) Immunolocalization of fibroblast growth factor-1 (FGF-1), its receptor (FGFR-1), and fibroblast-specific protein-1 (FSP-1) in inflammatory renal disease. Kidney International, 68, 2621-2628. http://dx.doi.org/10.1111/j.1523-1755.2005.00734.x

[13] Zittermann, S.I. and Issekutz, A.C. (2006) Basic fibroblast growth factor (bFGF, FGF-2) potentiates leukocyte recruitment to inflammation by enhancing endothelial adhesion molecule expression. American Journal of $\mathrm{Pa}$ thology, 168, 835-846.

http://dx.doi.org/10.2353/ajpath.2006.050479

[14] Andrés, G., Leali, D., Mitola, S., Coltrini, D., Camozzi, M., Corsini, M., Belleri, M., Hirsch, E., Schwendener, R.A., Christofori, G., Alcami, A. and Presta, M. (2009) A pro-inflammatory signatura mediates FGF2-induced an- 
giogenesis. Journal of Cellular and Molecular Medicine, 13, 2083-2108.

http://dx.doi.org/10.1111/j.1582-4934.2008.00415.x

[15] Strowig, T., Henao-Mejía, J., Elinav, E. and Flawell, R. (2012) Inflammosomes in health and disease. Nature, 481, 278-296. http://dx.doi.org/10.1038/nature10759

[16] Stewart, M.W. (2012) The expanding role of vascular endothelial growth factor inhibitors in ophthalmology. Mayo Clinic Proceedings, 87, 77-88. http://dx.doi.org/10.1016/j.mayocp.2011.10.001

[17] Lee, M., Kang, Y., Suk, K., Schwab, C., Yu, S. and McGeer, P.L. (2011) Acidic fibroblast growth factor (FGF) potentiates glial-mediated neurotoxicity by activating FGFR2 IIIb protein. Journal of Biological Chemistry, 286, 41230-41245. http://dx.doi.org/10.1074/jbc.M111.270470

[18] Aiello, L.P., Brucker, A.J., Chang, S., Cunningham Jr, E.T., D’Amico, D.J., Flynn Jr., H.W., Grillote, L.R., Hutcherson, S., Liebmann, J.M., O'Brien, T.P., Scout, I.U., Spaide, R.F., Ta, C. and Trese, M.T. (2004) Evolving guidelines for intravitreous injections. Retina, 24, S3S19.

http://dx.doi.org/10.1097/00006982-200410001-00002

[19] Verma, L., Sinha, R., Venkatesh, P. and Tewari, H.K. (2004) Comparative evaluation of diode laser versus argon laser photocoagulation in patients with central serous retinopathy: A pilot, randomized controlled trial [ISRCTN84128484]. BMC Ophthalmology, 4, 15. http://dx.doi.org/10.1186/1471-2415-4-15

[20] Sarks, J.P., Sarks, S.H. and Killingsworth, M.C. (1988) Evolution of geographic atrophy of the retinal pigment epithelium. Eye (Lond), 2, 552-577. http://dx.doi.org/10.1038/eye.1988.106

[21] Chappelow, A.V. and Kaiser, P.K. (2008) Neovascular age-related macular degeneration: Potential therapies. Drugs, 68, 1029-1036. http://dx.doi.org/10.1038/eye.1988.106

[22] Fernández, I.S., Cuevas, P., Angulo, J., López-Navajas, P., Canales-Mayordomo, A., Lozano, R.M., Valverde, S., Jiménez-Barbero, J., Romero, A. and Giménez-Gallego, G. (2010) Gentisic acid, a compound associated with plant defence and a metabolite of aspirin, heads a new class of in vivo FGF inhibitor. The Journal of Biological Chemistry, 285, 1714-1729. http://dx.doi.org/10.1074/jbc.M109.064618

[23] Cuevas, P., Outeiriño, L.A., Azanza, C. and GiménezGallego, G. (2012) Intravitreal dobesilate in the treatment of choroidal neovascularization associated with age-related macular degeneration. Report of two cases. British Medical Journal (BMJ) Case Reports.

[24] Cuevas, P., Outeiriño, L.A., Azanza, C., Angulo, J. and Giménez-Gallego, G. (2012) Short-term efficacy of intravitreal dobesilate in central serous chorioretinopathy. European Journal of Medical Research, 17, 22.
http://dx.doi.org/10.1186/2047-783X-17-22

[25] Cuevas, P., Outeiriño, L.A., Angulo, J. and GiménezGallego, G. (2012) Chronic cystoid macular oedema treated with intravitreal dobesilate. British Medical Journal (BMJ) Case Reports.

[26] Cuevas, P., Outeiriño, L.A., Angulo, J. and GiménezGallego, G. (2012) Treatment of dry age-related macular degeneration with dobesilate. British Medical Journal (BMJ) Case Reports.

[27] Cuevas, P., Outeiriño, L.A., Angulo, J. and GiménezGallego, G. (2012) Treatment of Stargardt disease with dobesilate. British Medical Journal (BMJ) Case Reports.

[28] Thomas, K.A. and Giménez-Gallego, G. (1986) Fibroblast growth factors: Broad spectrum mitogens with potent angiogenic activity. Trends in Biochemical Sciences, 11, 81-84.

http://dx.doi.org/10.1016/0968-0004(86)90271-9

[29] Cuevas, P., Carceller, F., Ortega, S., Zazo, M., Nieto, I. and Giménez-Gallego, G. (1991) Hypotensive activity of fibroblast growth factor. Science, 254, 1208-1210. http://dx.doi.org/10.1126/science.1957172

[30] Galan, J.M., Cuevas, B., Dujovny, N., Giménez-Gallego, G. and Cuevas, P. (1996) Sleep promoting effects of intravenously administered acidic fibroblast growth factor. Neurological Research, 18, 567-569.

[31] Cuevas, P., Angulo, J. and Giménez-Gallego, G. (2011) Topical treatment of contact dermatitis by pine processionary caterpillar. British Medical Journal (BMJ) Case Reports.

http://casereports.bmj.com/content/2011/bcr.06.2011.435 1.full.pdf

[32] Hanai, K., Oomura, Y., Kai, Y., Nishikawa, K., Shimizu, N., Morita, H. and Plata-Salamán, C.R. (1989) Central action of acidic fibroblast growth factor in feeding regulation. American Journal of Physiology, 256, R217-R223.

[33] Bame, K.J. (2001) Heparanases: Endoglycosidases that degrade heparan sulfate proteoglycans. Glycobiology, 11, 91R-98R. http://dx.doi.org/10.1093/glycob/11.6.91R

[34] Yuan, K., Hong, T.M., Chen, J.J., Tsai, W.H. and Lin, M.T. (2004) Syndecan-1 up-regulated by ephrinB2/EphB4 plays dual roles in inflammatory angiogenesis. Blood, 104, 1025-1033. http://dx.doi.org/10.1182/blood-2003-09-3334

[35] Chen, G., Wang, D., Vikramadithyan, R., Yagyu, H., Saxena, U., Pillarisetti, S. and Goldberg, I.J. (2004) Inflammatory cytokines and fatty acids regulate endothelial cell heparanase expression. Biochemistry, 43, 4971-4977. http://dx.doi.org/10.1021/bi0356552 


\section{ABBREVIATIONS}

BCVA: Best corrected visual acuity

SD-OCT: Spectral domain optical coherence tomography

FGF: Fibroblast growth factor

FGFR: Fibroblast growth factor receptors 